\title{
FIELD QUALITY AND MAGNETIC CENTER STABILITY ACHIEVED IN A VARIABLE PERMANENT MAGNET QUADRUPOLE FOR THE ILC
}

\author{
Y. Iwashita, ${ }^{\#}$ T. Mihara, Kyoto ICR, Uji, Kyoto \\ Masayuki Kumada, NIRS, Chiba-shi \\ Cherrill M. Spencer, SLAC, Menlo Park, CA, U.S.A.
}

\begin{abstract}
A superstrong permanent magnet quadrupole (PMQ) is one of the candidates for the final focus lens for the International Linear Collider (ILC). Our prototype PMQ can produce variable strengths from $3.5 \mathrm{~T}$ to $24.2 \mathrm{~T}$ in $1.4 \mathrm{~T}$ steps. The magnetic center of the PMQ must not move more than a few microns during a $20 \%$ strength change to enable a Beam-Based Alignment (BBA) process to work.

Our PMQ can be mechanically adjusted to suppress the center movement from more than $30 \mu \mathrm{m}$ to less than $10 \mu \mathrm{m}$ during strength changes.
\end{abstract}

\section{INTRODUCTION}

The precise tolerances on the last two quadrupoles before the interaction point of the proposed superconducting e+e- collider (ILC) have not been determined yet. These quads will be aligned with a Beam-Based Alignment (BBA) process during which their integrated strengths will be decreased by $20 \%$ [1]. Their magnetic centers must move by less than a few microns during the BBA else a systematic error will be introduced, yielding an increase in the beam spot size. These strong quads must be small to fit in the tight space. A compact, variable, superstrong permanent magnet quad (PMQ) has been fabricated and tested [2]. The PMQ has inner and outer rings of NEOMAX; the outer ring is subdivided along its length and each section can rotate. By rotating different lengths one can vary the integrated strength in small steps. Because of the fixed inner ring and tight mechanical tolerances, the sensitivities of the magnetic center and pole angles to the rotation of the outer rings are largely suppressed. Magnetic measurements of the PMQ are given. We show how observed, small, center and angle shifts were further reduced by adjustments to the stopping angles of the rotating rings and by shimming these rings.

\section{PERMANENT MAGNET QUADRUPOLE}

We fabricated a strength-variable permanent magnet quadrupole as a candidate for the final quad in a linear collider. The PMQ is composed of an inner ring and four outer rings [2]. (See Fig. 1 and table 1 for its dimensions and parameters.)

Only the outer rings are rotated in order to change the

\# mihara@kyticr.kuicr.kyoto-u.ac.jp

This research was partially supported by the Minsitry of Education, Science, Sports and Culture, Grant-in-Aid for Scientific Research (A), 14204023, 2002 and the U.S. Department of Energy under contract number DE-AC02-76SF00515. integrated gradient. The fixed inner ring suppresses any errors caused by rotation of outer rings. Outer rings are rotated just $90^{\circ}$ to switch the phase of that section's quadrupole (from focus to defocus and vice versa).

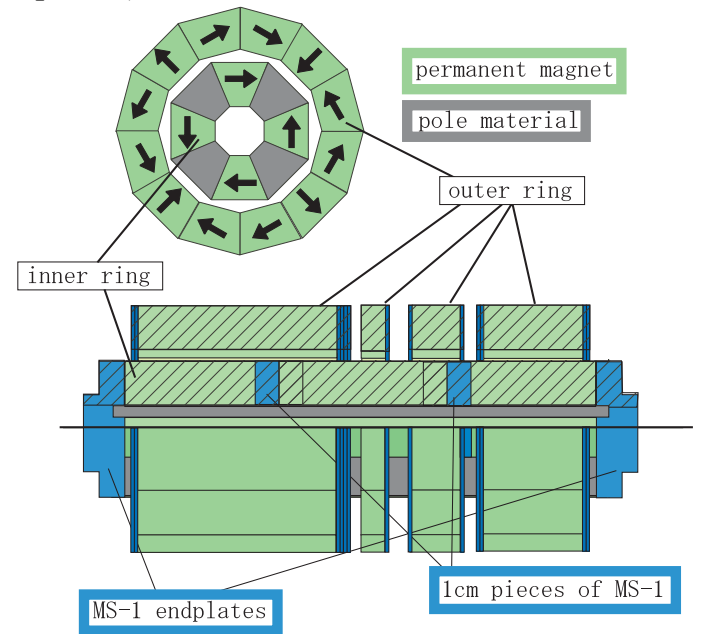

Figure 1: Fabricated Adjustable PMQ.

Table 1: Scale and performance of the PMQ

\begin{tabular}{|l|l|}
\hline Bore radius & $1 \mathrm{~cm}$ \\
\hline Inner ring radii & In $1 \mathrm{~cm}$ out $3 \mathrm{~cm}$ \\
\hline Outer ring radii & In $3.3 \mathrm{~cm}$ out $5 \mathrm{~cm}$ \\
\hline Outer ring section lengths & $1 \mathrm{~cm}, 2 \mathrm{~cm}, 4 \mathrm{~cm}, 8 \mathrm{~cm}$ \\
\hline Physical length & $23 \mathrm{~cm}$ \\
\hline Pole material & Permendur \\
\hline Magnet material(inner ring) & NEOMAX38AH \\
\hline Magnet material(outer ring) & NEOMAX44H \\
\hline Integrated gradient(strongest) & $24.2 \mathrm{~T}(25.2 \mathrm{~T})$ \\
\hline Integrated gradient(weakest) & $3.7 \mathrm{~T}(4.5 \mathrm{~T})$ \\
\hline Int. gradient step size & $1.4 \mathrm{~T}$ \\
\hline
\end{tabular}

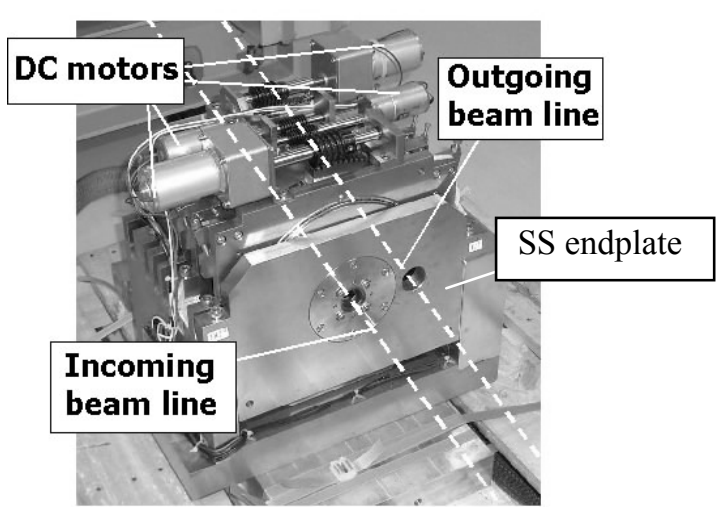

Figure 2: Fabricated adjustable PMQ. 
Therefore, this PMQ with four outer rings can produce a series of 16 integrated gradients from $3.7 \mathrm{~T}$ to $24.2 \mathrm{~T}$ in $1.4 \mathrm{~T}$ steps. Integrated gradient is proportional to "Switched on" length (SWL), the summation of outer rings lengths which are at the "strong" phase [2].

The inner ring is directly supported by stainless steel (SS) end plates that are fixed on the magnet base block. Each outer ring is supported in a ball bearing that is fitted into a SS ring support plate. Each outer ring has a spur gear that is rotated (4 DC motors with worm gears move the spur gears). These four plates are secured on the SS base block.

\section{FIELD QUALITY}

The higher multipole components of the PMQ should be within the tolerances of the ILC FF quad, but they have not been specified yet. The multipole components of the PMQ have been measured at two different strengths (see Fig.3) by a "double-coil" rotating coil that over-estimates the size of the sextupole component $(n=3)$. We do not believe the sextupole/quad is much more than $1 \times 10^{-3}$ Left graph in Fig. 3 shows multipoles measured at $4 \mathrm{~mm}$ at $100 \%$ and $55 \%$ strength, they are almost the same, which is preferred.

Right graph in Fig. 3 shows how little the multipole components change when the $8 \mathrm{~cm}$ ring is shimmed in
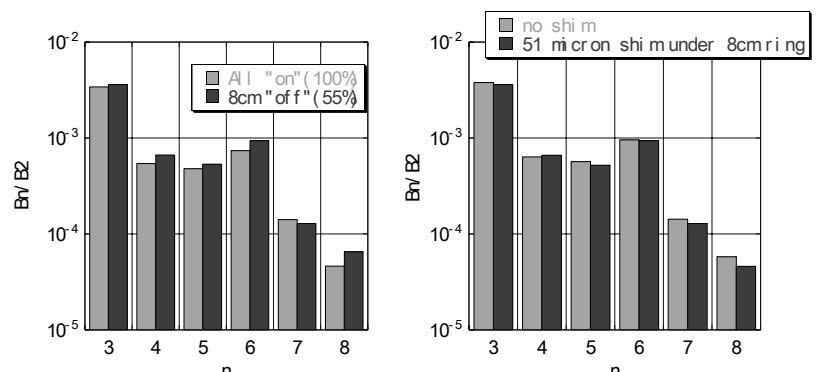

Figure 3: Measured Multipole Components at $4 \mathrm{~mm}$. Left: $100 \%$ strength (all on) \& 55\% (8 cm off) Right: $8 \mathrm{~cm}$ ring shimmed or not with $8 \mathrm{~cm}$ off.

order to adjust the magnetic center (see below).

\section{POLE ANGLE ADJUSTMENT}

It is necessary to adjust the stopping angle of each outer ring precisely because the skew angle of the ILC's final quad's field must be less than a few $\mu \mathrm{rad}$. The procedure for adjusting the stop angles and the results follow.

The stopper block fixed on the spur gear comes in contact with the stopper lever which is fixed on the ring support plate, and the rotation stops. Therefore, the rotation stop angle of an outer ring is controllable by the stopper lever position; it can be fine-tuned by turning the appropriate thrust screw (see Fig. 4).

If the phase of the inner ring's quad equals that of the outer ring, the quadrupole strength should reach a maximum. We tuned the stopper by turning the screw, checking the integrated strength by a rotating coil measurement system, and searched for the best position.
It is thought that the angle that gives the maximum (minimum) value of the magnetic field is the correct stop ping angle $(\mathrm{skew}=0)$. For instance, figure 5 shows that the phases of the inner ring and the $8 \mathrm{~cm}$ outer ring become completely the same when the angle of PMQ is about $43.715^{\circ}$ (a relative angle value of the $1^{\text {st }}$ south pole).

Fig. 5 shows that the skewing of the quadrupole angle is suppressed on the PMQ. The screw proceeds $1 \mathrm{~mm}$ a turn and the stopper lever move a few hundred microns. A turn of screw corresponds to about $30 \mu \mathrm{rad}$ on the figure 4 . So about $3 \mathrm{mrad}$ error in the rotation of the $8 \mathrm{~cm}$ outer ring causes only a $20 \mu \mathrm{rad}$ skew in the quadrupole angle of the PMQ.

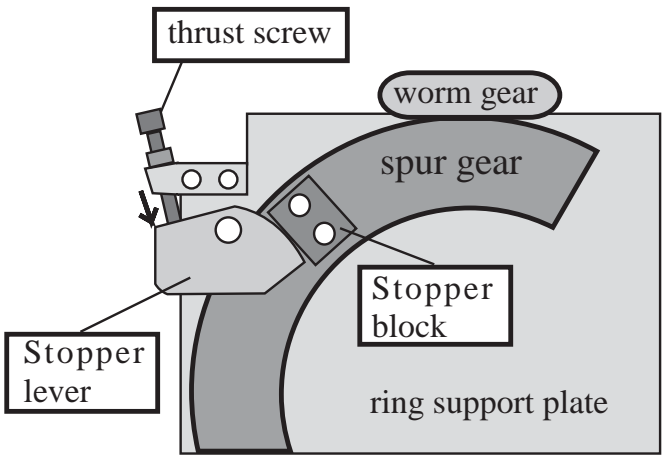

Figure 4: Gear stopper adjustment. Stop angle on each side can be adjusted by the screw.

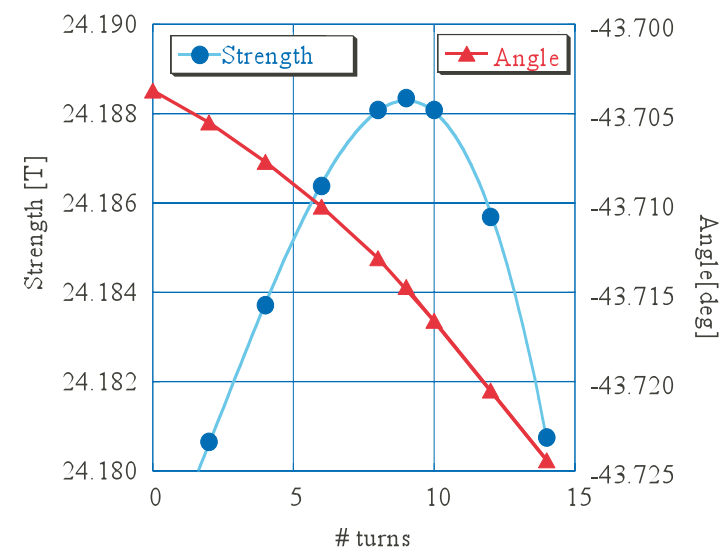

Figure 5 : Angle adjustment of $8 \mathrm{~cm}$ outer ring.

\section{MAGNETIC CENTER ADJUSTMENT}

The good operation of the ILC depends on successful $\mathrm{BBA}$, which requires the final quad's placement to be correct to within a few microns. Which means the magnetic center of the PMQ must move less than a few microns when an outer ring is rotated to change its strength.

When the PMQ was first assembled and measured we observed that its magnetic center position, defined by $\mathrm{X}$ and $\mathrm{Y}$ coordinates quoted relative to the axis of the rotating measuring coil, moved by several tens of microns when measured at different strengths. (See Fig. 6) There are two scenarios that could produce a center movement. 


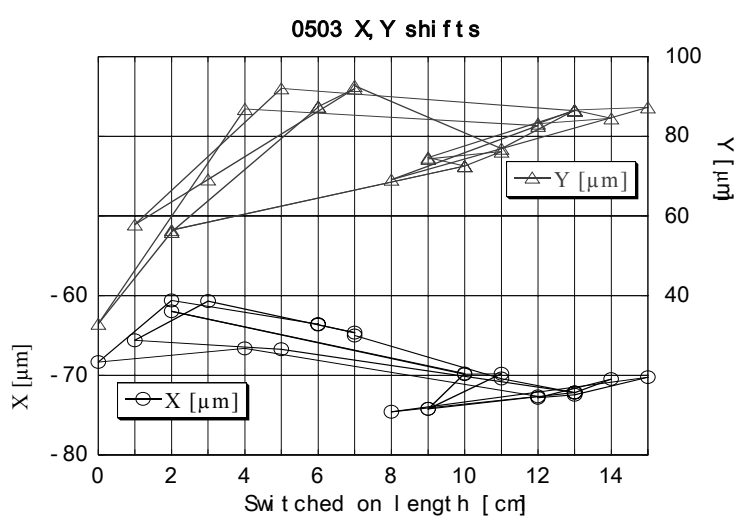

Figure 6: Center position of PMQ at different strengths. Lines between points show history of measurements.

First is that the magnetic center axes of the individual outer rings do not coincide with their rotation axes. Second is that the rotation axis of an outer ring is shifted from the magnetic axis of the inner ring. These two scenarios affect the $\mathrm{X}$ and $\mathrm{Y}$ movements differently, we measure the overall result when we change the strength. The rings were fabricated and assembled together to very tight tolerances. There are ways of shifting the relative positions of the rings.

The inner ring is fixed directly to the SS base in our PMQ, and each outer ring is supported by a bearing fitted in that ring's support plate. Each plate with an outer ring is put on the SS base block, and fixed with screws.

It is possible to shift an outer ring by placing a shim between a ring support plate and the SS base block where the outer ring is supported. (See Fig.7) We put different thicknesses of shims between the SS base block and the 4 outer ring cases, thus moving each outer ring, one at a time, and measured the movement of the magnetic center. Shimming can only lift up or move an outer ring in the $-X$ direction, therefore we could not adjust the center in the $-\mathrm{Y}$ direction or $+\mathrm{X}$ direction. Grinding the $\mathrm{SS}$ frame or SS base would make it possible to adjust all outer rings and shift the center in any direction.

Lifting the $8 \mathrm{~cm}$ outer ring up by $51 \mu \mathrm{m}$ reduced the center movement from $30 \mu \mathrm{m}$ to $10 \mu \mathrm{m}$ when the strength

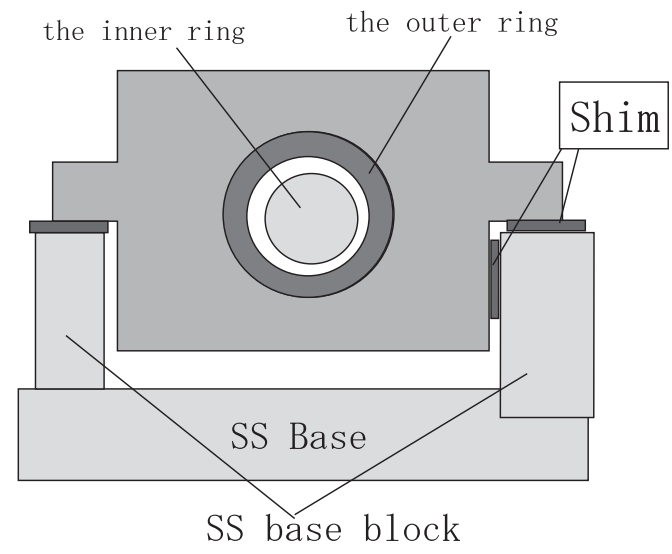

Figure 7: Sketch of PMQ showing shim placement.
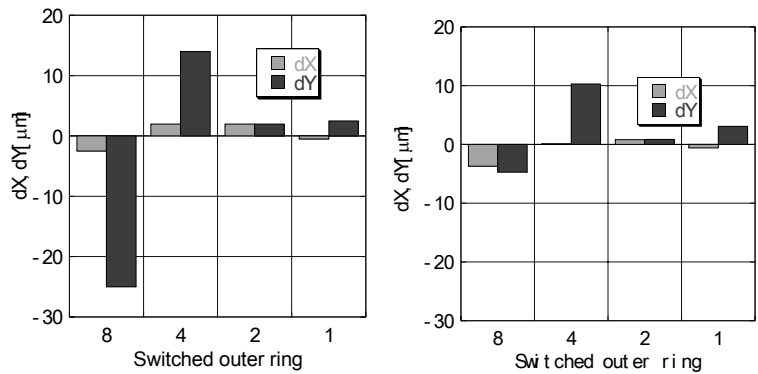

Figure 8: Center Movement affected by shimming. Left: Before shimming. Right: $8 \mathrm{~cm}$ ring lifted up by $76 \mu \mathrm{m}$. " 8 " means $8 \mathrm{~cm}$ long outer ring.

was changed by about $45 \%$ (see Fig. 8 left). Fig. 8 right shows an example of shimming by lifting and horizontal displacement. The $8 \mathrm{~cm}$ outer ring is lifted up by $76 \mu \mathrm{m}$ and $4 \mathrm{~cm}$ outer ring is moved to $-\mathrm{x}$ direction by $51 \mu \mathrm{m}$. Then the Y-magnetic center shift when $8 \mathrm{~cm}$ is rotated becomes $<5 \mu \mathrm{m}$ and the $\mathrm{X}$-shift of $4 \mathrm{~cm}$ outer ring becomes $<1 \mu \mathrm{m}$.

\section{CONCLUSIONS}

The PMQ's field quality is moderately good and it was not affected by changing integrated strength or shimming.

The control of the stopping angle was effected by adjusting the stopper; it decreased the overall rotation of PMQ. One turn of screw on $8 \mathrm{~cm}$ outer ring stopper adjusts about $20 \mu \mathrm{rad}$ on this PMQ at strongest case.

The magnetic center axes of the outer rings are adjusted by using $25.4 \mu \mathrm{m}$ shims. The center movement caused by rotation of the $8 \mathrm{~cm}$ outer ring was successfully reduced from some tens of microns to $5 \mu \mathrm{m}$ in $\mathrm{Y}$ coord by lifting it up by $76 \mu \mathrm{m}$. The effect of the $4 \mathrm{~cm}$ outer ring on the $\mathrm{X}$ coord could also be reduced by moving it in the $-\mathrm{X}$ direction by $51 \mu \mathrm{m}$.

Because the shimming worked very well in some cases, an automated shimming mechanism should be incorporated in a future design. Particularly, fine adjustments in both polarities are important.

Residual center movements after these adjustments indicate that the symmetries of the outer rings are not perfect. Shimming or shifting mechanisms for the outer rings against their bearings should guarantee this design of a quad will meet the tight tolerances on the magnetic center behavior of the final quad in the ILC.

\section{REFERENCES}

[1] P. Tenenbaum, et.al. "Beam Based Alignment of the Final Focus Test Beam" SLAC-PUB-7058, Dec 1995.

[2] T. Mihara , Y. Iwashita, M.Kumada, C.M.Spencer "Superstrong Adjustable Permanent Magnet for a Linear Collider Final Focus" $12^{\text {th }}$ Linear Accelerator Conference. Lubeck, Germany. August 16-20, 2004. SLAC-PUB-10878. 